\title{
CTEQ Parton Distributions and Flavor Dependence of Sea Quarks*
}

\author{
James Botts ${ }^{a}$, Jorge G. Morfin ${ }^{b}$, Joseph F. Owens ${ }^{c}$, Jianwei Qiu ${ }^{d}$, \\ $\mathrm{Wu}-\mathrm{Ki}$ Tung $^{a}$ and Harry Weerts ${ }^{a}$ \\ ${ }^{a}$ Michigan State University, \\ ${ }^{b}$ Fermi National Accelerator Laboratory, \\ ${ }^{c}$ Florida State University, ${ }^{d}$ Iowa State University
}

(to be published in Physics Letters)

\begin{abstract}
This paper describes salient features of new sets of parton distributions obtained by the CTEQ Collaboration円 based on a comprehensive QCD global analysis of all available data. The accuracy of the new data on deep inelastic scattering structure functions obtained by the very high statistics NMC and CCFR experiments provides unprecedented sensitivity to the flavor dependence of the sea-quark distributions. In addition to much better determination of the small $x$ dependence of all parton distributions, we found: (i) the strange quark distribution is much softer than the non-strange sea quarks and rises above the latter at small- $x$; and (ii) the difference $\bar{d}-\bar{u}$ changes sign as a function of $x$. A few alternative sets of viable distributions with conventional assumptions are also discussed.
\end{abstract}

*The CTEQ Collaboration is funded by Texas National Laboratory Commission. This work is also partially supported by NSF and DOE through grants made to the home institutions of the authors.

${ }^{1}$ CTEQ is an acronym for Coordinated Theoretical/Experimental Project on QCD Phenomenology and Tests of the Standard Model. The Collaboration consists of, in addition to the above authors (as members of its global fit subgroup), R. Brock, J. Huston, J. Pumplin, C.P. Yuan (MSU); J. Collins, J. Whitmore (PSU); S. Kuhlmann (Argonne); S. Mishra (Harvard); F. Olness (SMU); D. Soper (Oregon); J. Smith, and G. Sterman (Stony Brook). 
Introduction In the current theoretical framework, high energy lepton-hadron and hadron-hadron interaction cross-sections $\sigma$, both in standard model and in new physics processes, are related to calculable fundamental parton interaction cross-sections $\widehat{\sigma}$ by the QCD factorization theorems [1] as a sum of integrals convoluting the latter with universal parton distribution functions. The parton distributions can, in principle, be determined from analyzing a set of standard experiments — deep inelastic scattering (DIS), leptonpair production (LPP), high- $p_{t}$ direct-photon production, W- and Z-production, high- $p_{t}$ jet-production, heavy flavor production, ... etc. As both theory and experiments have matured and grown in complexity and as the scope of the standard experiments expands to various collider processes, the task of proper and systematic global QCD analyses requires coordinated efforts of both theorists and experimentalists familiar with details of both sides of such an analysis. For this reason, the CTEQ Collaboration has been developing the necessary tools for carrying out up-to-date global analyses based on previous work of Duke-Owens [2] and Morfin-Tung[3].

Previous global analyses were based on data from the SLAC-MIT, EMC, CDHSW, and BCDMS deep inelastic scattering experiments; the E288, E605 lepton-pair production experiments; and the WA70 direct photon production experiment. [4] Recently released NMC data[5] on $F_{2}^{n} / F_{2}^{p}, F_{2}^{p}-F_{2}^{n}$, and $F_{2}^{p, d}$ using a muon beam and CCFR data[6] on $F_{2,3}^{F e}$ using (anti-) neutrinos are expected to have a significant impact on QCD global analyses because of their extended kinematic coverage (particularly at small $x$ ), their high statistics and minimal systematic errors. The precision of the current generation of DIS experiments now far exceeds the size of next-to-leading order QCD contributions to these processes; thus they probe the full complexity of QCD mixing effects between quarks and gluons in a properly conducted QCD analysis. We find that these new accurate DIS data, supplemented by constraints imposed by data on lepton-pair and direct photon production, leads to substantially increased sensitivity to the flavor content of the seaquark distributions as well as to the gluon distribution — features conventionally thought to be beyond the reach of analyses involving totally inclusive data.

To demonstrate the need for new comprehensive global analyses and the potential for discovering new features of parton distributions in light of the new data, we show in table I values of $\chi^{2}$ per degree of freedom obtained by comparison of old and new data with theoretical numbers obtained from parton distributions widely used in the current literature: KMRS[7], MT-S1, MT-B2]3], and the recent MRS D0 8] (which is based on earlier preliminary NMC and CCFR data). We note that all old sets do rather badly with the new data. Only the MT-B2 set is close to being acceptable, but still misses the CCFR data by 1.5 - 2 standard deviations. Even the MRS D0 set (which used the new data as input) does poorly, with most of its high $\chi^{2}$ also coming from the CCFR data.2]

\footnotetext{
${ }^{2}$ These large $\chi^{2}$ values are consistent with the original MRS analysis because the errors were enlarged in that analysis.
} 
General Description of New Analysis For the current analysis, we use the following sets of data: BCDMS $F_{2}^{\mu p} \& F_{2}^{\mu d}$, NMC $F_{2}^{\mu p}, F_{2}^{\mu d} \& F_{2}^{\mu n} / F_{2}^{\mu p}$, [CCFR $F_{2}^{\nu F e} \& F_{3}^{\nu F e}$ DIS structure functions, E605 LPP $d \sigma / d y d \tau$, and WA70, E706 \& UA6 direct photon $d \sigma / d y d p_{t}$. With kinematic cuts $\left(Q^{2}>4 \mathrm{GeV}^{2}\right.$ in DIS and LPP, $W^{2}>12 \mathrm{GeV}^{2}$ in DIS, and $p_{t}>$ $4 \mathrm{GeV}$ in direct photon production), the total number of data points used in most of our analysis is 917 . We have tried higher $Q^{2}$-cuts and found the results are not sensitive to the choice. For the very high-statistics DIS experiments, statistical errors have been reduced to such a low level that systematic errors often dominate the experimental uncertainties. The systematic errors clearly cannot be neglected as has been done in some past analyses. On the other hand, a complete treatment of these errors, including all relevant correlations (as is usually done in single-experiment analyses), would be (a) entirely impractical in the context of a large-scale global analysis, and (b) uncertain in statistical significance since such a diverse set of experimental data is involved. Thus, we adopt the common practice of combining the statistical and systematic errors in quadrature point-to-point. We have also tried to add the errors linearly, and found similar conclusions (with, of course, reduced absolute values of $\chi^{2}$ ). The relative normalization of the various data sets is allowed to vary around 1.0 during the fitting process with an associated $\chi^{2}$ included using the appropriate quoted experimental normalization errors. Heavy target corrections are applied to the neutrino structure functions to generate equivalent isoscalar structure functions for nucleons. The correction factor is taken from measured ratios of structure functions on light to heavy targets measured in muon scattering experiments. [9] In the crucial low-x region, the default correction factor taken for this analysis is a conservative factor based on the assumption of saturation of the shadowing effect for A $>40$. Since this correction is significant compared to current experimental errors, we have also investigated changes resulting from using a correction factor based on a fit of shadowing data off He, $\mathrm{C}$ and Ca with subsequent extrapolation to Fe. The differences will be noted later.

We perform least $\chi^{2}$ fits using primarily the MINUIT program. All results have been verified and investigated by a completely independent program package developed by Duke and Owens. [2] We use the overall $\chi^{2}$ as well as individual $\chi^{2}$ 's for each experiment as measures of the relative "goodness of fit", but do not attach strict statistical significance to the absolute values of the $\chi^{2}$ 's for reasons mentioned above. For quantitative nextto-leading order (NLO) QCD global analyses based on data of high accuracy, one must take into account a number of non-trivial theoretical and experimental considerations. These issues have been described in detail in several reviews, [4], [10], [1] hence will not be discussed here.

\footnotetext{
${ }^{3}$ Although the ratio measurement is not totally independent from the individual $F_{2}^{\mu p} \& F_{2}^{\mu d}$, it is in fact more significant than the latter since it involves considerably smaller systematic errors and a somewhat extended kinematic range; hence this data set is included upon the advice of some members of the NMC collaboration.
} 
Recent experiments have called into question a number of traditional assumptions about the unknown input parton distributions (at some fixed scale $Q_{0}^{2}$ ) such as $\mathrm{SU}(3)$ or $\mathrm{SU}(2)$ flavor symmetry for the sea-quarks. Since the diversity of data sets used in the analysis and the measurement accuracy have increased sufficiently to provide stringent constraints on the theoretical unknowns, we find it desirable to try fits which are free from most conventional prejudices and compare the results with those obtained with more traditional assumptions. This comparison turns out to be very revealing.

For most of our analyses, the QCD evolution kernel, the effective coupling, and all hard scattering cross-sections are taken to be in the $\overline{\mathrm{MS}}$ renormalization scheme; hence the extracted parton distributions are, by definition, in the $\overline{\mathrm{MS}}$ scheme. For the convenience of certain applications, we also obtained parton distribution functions defined in the DIS scheme. These distributions are generated by independent fits to the same data.f Likewise, we generate representative leading-order fits for applications which use LO hard matrix elements. For concreteness, in the following description of our results, we shall focus on the $\overline{\mathrm{MS}}$ distributions.

Description of Results The most notable fact of this new global analysis is the extraordinary quantitative agreement of the NLO-QCD parton framework with the very high statistics DIS experiments over the entire kinematic range these experiments cover and the consistency of this framework with all available experiments in lepton pair and direct photon production as well. In the least constrained fits, the overall $\chi^{2}$ is typically around 860 for about 880 degrees of freedom (917 data points with $30 \sim 35$ parton shape and relative experimental normalization fitting parameters); and the $\chi^{2}$ for the individual experiments are all comparable to the respective number of points - a remarkable result considering the diversity of processes, the kinematic range covered, and the accuracy of many of the experiments. Other fits with certain restrictions (such as $\mathrm{SU}(2)$ or $\mathrm{SU}(3)$ symmetric sea) have overall $\chi^{2}$ around 910 or higher. Although the relative values of $\chi^{2}$ are considerably larger than the best fit, even these alternatives can represent reasonable fits when taken by themselves. The plots of these fits against data (not included in this letter) give the impression of good agreement in most cases and only very careful examination will reveal the differences. The merit of these possible solutions can be better judged by examining details of the parton distributions obtained and of the comparison with the various data sets used, as will be discussed later.

In general we find two common features of the new fits:

(i) The small- $x(\sim 0.01-0.08)$ data from NMC and CCFR require substantially increased sea-quark distributions in this region. We found this also produces an indirect effect on

\footnotetext{
${ }^{4}$ This is a more reliable procedure then by transforming from the $\overline{\mathrm{MS}}$ sets since the "NLO" term in the conversion formula can, under some circumstances, be comparable in size to the "LO" term, thus vitiating the perturbative nature of the transformation. 3.
} 
the shape of the gluon distribution such that the momentum fraction carried by the gluon at $Q=2 \mathrm{GeV}$ is reduced from the previously accepted value $\sim 0.45$ to around 0.42 . This fraction is partially recovered after evolution to larger $Q$. (Note that in NLO QCD the gluon momentum fraction is factorization scheme dependent (i.e. different in the $\overline{\mathrm{MS}}$ and DIS schemes); and it is not exactly tied to the integral of the neutrino structure function $F_{2}$ as in leading order.)

(ii) As a free-parameter in the global fit, the NLO $\Lambda_{Q C D}(5$ flavors $)$ is found to be typically 150 to $160 \mathrm{MeV}$ in these new fits - consistent with typical values $\left[\Lambda_{Q C D}(4 f l)=\right.$ 220 to $240 \mathrm{MeV}$ ] found in direct $d \ln F_{2} / d \ln Q^{2}$ analyses [12] and in previous global analyses.[3], 13] Fits with $\Lambda_{Q C D}(5 \mathrm{fl})$ fixed at $220 \mathrm{MeV}$ (corresponding to a higher value of $\alpha_{s}$ favored by some LEP measurements) have substantially higher $\chi^{2}$ — by about 30 .

In Table II we present a set of representative fits with different characteristics and list their overall $\chi^{2}$ as well as the $\chi^{2}$ per data points for the various data sets. The best fit in the $\overline{\mathrm{MS}}$ scheme, with the least constraints, is designated CTEQ1M. For this set, the input distribution functions at $Q_{0}(=2 \mathrm{GeV})$ for all the flavors are taken to be of the form: $f\left(x, Q_{0}\right)=A_{0} x^{A_{1}}(1-x)^{A_{2}}\left(1+A_{3} x^{A_{4}}\right)$. The coefficients $A_{i}$ are subjected to minimal restrictions in addition to the quark-number and momentum sum rules. Although, it may appear that there are many parameters to be determined, it turns out that the wealth of data provides surprisingly stable results for the best fit irrespective of where the starting point is taken to be in the parameter space. A glance across the first row of Table II reveals that all data sets are uniformly well-fit】, indicating a good deal of consistency. The most conspicuous feature of the CTEQ1M distributions is the rather large flavor dependence of the sea quarks - the $\bar{u}, \bar{d}$, and $\bar{s}$ distributions all differ substantially in shape. See Fig.1. In addition, because of the significantly increased $s(x)$ in the small- $x$ region, the normalization of the strange quark, as measured by the ratio of momentum fractions (integrated over the full $x$-range $(0,1)) \kappa=2 s /(\bar{u}+\bar{d})$, is around 0.9 instead of the often quoted value of 0.5.[14] Since these results appear to be unusual, we will discuss them in some detail after we describe the other fits in order to provide a basis for comparison.

CTEQ1D and CTEQ1L are DIS and LO distributions with the same assumptions as CTEQ1M. The DIS distribution gives equally good fit to data as the $\overline{\mathrm{MS}}$ one, just as one

\footnotetext{
${ }^{5}$ This result supersedes a preliminary CTEQ value reported at the 1992 International High Energy Conference at Dallas 10 which is inaccurate due to a computer error.

${ }^{6}$ We adopt the following label scheme for our parton distributions: CTEQnSX, where $\mathrm{n}$ is a version number; $\mathrm{S}$ designates the factorization scheme $-\mathrm{M}$ for $\overline{\mathrm{MS}}$, D for DIS, and $\mathrm{L}$ for $\mathrm{LO}$; $\mathrm{X}$ is absent for the best fits, otherwise it distinguishes the alternative sets within a given scheme. Parametrizations of these distributions can be obtained by sending a request to Botts@MSUPA (bitnet) or Botts@MSUPA.PA.MSU.EDU (internet).

${ }^{7}$ The only apparently "high" $\chi^{2}$ associated with the NMC hydrogen date is due to differences of the NMC and BCDMS data around the $x=0.225$ bin where the more abundant and more accurate BCDMS data dominate the fit.
} 
expects - a consistent change in factorization scheme should not change the physics. The LO fit has a substantially higher $\chi^{2}$ (by $\sim 50$ ) and associated normalization correction factor for the LPP data, confirming the need of the NLO formalism for precision QCD analyses. CTEQ1MS is a set of distributions with a "singular" gluon behaving like $x^{-0.5}$ at $Q_{0}$; its $\chi^{2}$, comparable to the best fits, indicates that current data allow a range of different extrapolations to smaller $x$ beyond 0.01. And CTEQ1ML is a set obtained with $\Lambda(5 f l)$ fixed at $220 \mathrm{MeV}$ (the "LEP event shape value"). All these distributions give reasonable fits to data with different characteristics (and some with associated limitations, such as the LO one). We shall not discuss them further in this short report.

The row labeled M2 represents a fit assuming a SU(2) flavor symmetric sea. The overall $\chi^{2}$ is higher than that of CTEQ1M by 50, all data sets are uniformly well-fit as for the latter. However, it is associated with a normalization of the strange quark corresponding to $\kappa=1.41$ - too high a value from the physics point of view. In order to investigate the sensitivity of the combined data set to the strange quark normalization and shape, several fits with $\kappa$ fixed at 0.5 (according to conventional wisdom) are tried. The last row of Table II, labeled K5, represents the best fit one can get with this restriction. The overall $\chi^{2}$ increased dramatically by 88 . If one requires the shape of $s\left(x, Q_{0}\right)$ to be the same as the non-strange sea distributions, the $\chi^{2}$ also increases very substantially. Thus, contrary to conventional expectations, we found this data set (consisting of only totally inclusive quantities) is sensitive to $\kappa$ and to the shape of $s\left(x, Q_{0}\right)$, and it strongly prefers $\kappa \approx 0.9$ or higher.

To trace the origin of this surprising result, we note the principal difference between the CTEQ1M and the K5-fits in Table II is the increased $\chi^{2}$ values of the CCFR $F_{2}^{\nu}$ and the NMC $F_{2}^{\mu d}$ data sets. In leading order QCD, $F_{2}^{\nu d}$ (converted from $F_{2}^{\nu F e}$ using a heavy-target correction factor) is given by the straight sum of all quark flavors whereas $F_{2}^{\mu d}$ corresponds to the same sum weighted by the squared charges. One can therefore get a direct handle on the strange quark distribution by examining the equality $\frac{5}{6} F_{2}^{\nu d}-3 F_{2}^{\mu d}=x s(x, Q)+O\left(\alpha_{s}\right)$, valid at small $Q$ where the charm distribution is small. Fig.2 shows a plot of the quantity on the left-hand side of this equation at $Q^{2}=5 \mathrm{GeV}^{2}$ using data from NMC and CCFR, and compares it with the strange quark distributions of CTEQ1M, K5, and MRS D0 at the same $Q^{2}$. The size of the experimental errors are superimposed on the $\frac{5}{6} F_{2}^{\nu d}-3 F_{2}^{\mu d}$ curve. It is clear from this plot that $\kappa \simeq 0.5$ parton distribution sets are not compatible with the new data; and that both the size and the shape of $s(x, Q)$ in the best fit are driven directly by data. How can this conclusion be reconciled with the conventional low value of $\kappa$ obtained from leading order parton model analysis of dimuon production data? 14 First, we note that our $\kappa$ is defined at a fixed $Q^{2}\left(=4 \mathrm{GeV}^{2}\right)$ and is integrated over the full $x$-range $(0,1)$; whereas the $\kappa$ determined from the dimuon experiments use data from a wide range of $Q^{2}$ extending from less than $1 \mathrm{GeV}^{2}$ for the lowest $x$ values to well over $100 \mathrm{GeV}^{2}$ at moderate values of $x$. The two quantities are not the same as 
$\kappa$ is $Q$-dependent for a non-SU(3) symmetric sea. Secondly, from Fig.1, we see that for $x>0.1$ our $s(x, Q)$ actually becomes considerably smaller then $\bar{u}$ and $\bar{d}$ as one expected. If $\kappa\left(x_{\mathrm{min}}\right)$ is defined as the ratio of momentum fractions integrated from some $x_{\text {min }}$ to 1 , then $\kappa(0.1) \approx 0.5$; it increases to $\kappa(0.01) \approx 0.85$ and eventually to $\approx 0.9$ in the theoretical limit $x_{\text {min }} \rightarrow 0$. Thus, the comparison with the dimuon experiment is also dependent on the effective $x$-range and the extrapolations used in going beyond that range. Finally, as has been pointed out, 15 existing LO dimuon analyses are likely to be unreliable at small $x$ due to the hitherto neglected contribution from the gluon-fusion mechanism which is nominally next-to-leading order but physically significant.

The question of $\mathrm{SU}(2)$ symmetry of the non-strange sea can be investigated by comparing the two fits CTEQ1M (non-symmetric, $\kappa=0.9$ ) and M2 (symmetric, $\kappa=1.4$ ) in Table II. The increase in the overall $\chi^{2}$ of 52 mainly comes from the CCFR $F_{2,3}$ and E605 data sets. We know that the $p N$ LPP cross-section should be sensitive to $\bar{u}$ because of the dominance of $u \bar{u}$ scattering. $\underbrace{3}$ When restrictions are imposed on the flavor dependence of the sea, it is the interplay between the CCFR, NMC and E605 data sets which causes the $\chi^{2}$ to increase. While M2 has an (arguably) acceptable $\chi^{2}$, it does have an abnormally large strange quark component. If one restricts the size of $s\left(x, Q_{0}\right)$, say $\kappa \leq 1.0$, any solution with an SU(2) symmetric sea will have an unacceptable high $\chi^{2}$. We also note, in the preferred set, CTEQ1M, the $\bar{u}$ and $\bar{d}$ distributions cross each other around $x \sim 0.06$. This behavior contrasts with that of MRS D0 where $(\bar{d}-\bar{u})$ is chosen to be positive definite. We have found that the behavior shown is preferred for all the fits we have tried whenever the sign of this difference is left free. In In this connection, we mention there is some tantalizing experimental evidence for this behavior of $(\bar{d}-\bar{u})$ from E772 at Fermilab.[16] We know of no reason why this quantity need be positive definite.

Details on the above mentioned fits will be described in a full-length paper.

Uncertainties and Challenges There are uncertainties associated with this analysis some of which can be addressed. Concerning the heavy target correction in the small- $x$ region, we found the use of alternative shadowing correction schemes (cf. Sec.2) leads to larger upward corrections to $F_{2}^{\nu d}$, hence an even bigger strange-sea. One may also consider the effect of shadowing inside deuterium on relating deuterium to nucleon structure functions used in the analysis. Recent theoretical studies indicate that this effect is less than 2-3\% at the smallest- $x$ value covered here. [17] This is small compared to the uncertainties of the heavy-target correction. Detailed work can be done to quantify both these uncertainties. We also note, current analyses of DIS neutrino structure functions use the NLO QCD formalism for massless quarks, including the small but non-negligible

\footnotetext{
${ }^{8}$ The CTEQ analysis uses the full E605 data set on $d \sigma / d y d \tau$ (120 points); whereas, it appears from the published literature that, the MRS analyses use only the integrated $d \sigma / d \tau$ data for consistency checks [13.

${ }^{9}$ This may be partially responsible for the very high $\chi^{2}$ values on the CCFR data using the MRS D0 distributions (cf. Table I).
} 
$s \rightarrow c$ contribution. The effect of the charm quark mass is taken into account by applying a "charm-threshold correction" to the data, not at the theoretical end. This is not fully satisfactory because the applied "correction" is, at best, a leading order one. Since the complete NLO theory for this transition now exists, [18] the correct procedure is to include this in the theoretical expression and to compare with the uncorrected physical structure functions. We do not, however, expect either of these possible improvements to affect the main features of our results since, as we have demonstrated, they are driven rather directly by the new data. The only alternatives to the large strange sea at small-x would be: (i) there are additional unspecified systematic errors associated with the two major experiments at small-x; and (ii) the theoretical corrections due to charm-mass effects are much larger than expected and turn out to account for the bulk of the observed difference $\frac{5}{6} F_{2}^{\nu d}-3 F_{2}^{\mu d}$ in place of $x s(x, Q)$. The last possibility is under investigation.

Hadron collider data on W-, Z-production, lepton pair production, direct-photon production, jet production, and heavy flavor production are providing useful tests of the conventional QCD calculations. The expected increase in integrated luminosity by an order of magnitude at the Tevatron will make these measurements important sources of quantitative information on parton distributions. The new parton distributions obtained in our global analysis lead to increased cross-section for all the above mentioned collider processes in the regions dominated by small- $x$ partons (low mass lepton pair, low $p_{t}$ direct photon, and heavy flavor production). This is gratifying since earlier parton distributions tend to give smaller cross-sections on all these processes when compared to preliminary CDF data. Input from new results obtained in the current run at the Tevatron will help probe the very small- $x$ region; and will measure, in addition, different combinations of parton distributions than currently available. Given the tight constraints on these functions already found in this analysis, the new input will certainly provide significant quantitative tests of the consistency of the QCD parton framework and lead to even better determination of the parton distributions. We also, of course, eagerly anticipate results from the HERA ep collider 19 which will provide a wealth of information on structure functions and other observables sensitive to parton distributions in a widely expanded kinematic range.

Acknowledgment We would like to thank our colleagues on the CTEQ Collaboration for consultation and for encouragement. We would also like to thank Alan Martin and Dick Roberts for useful communications.

\section{Note Added in Proof:}

Since this paper was submitted, a new leading-order QCD analysis of dimuon events in neutrino scattering has been published by the CCFR group (PRL 70, 134 (1993)), 
reaffirming a smaller strange sea than the non-strange one. The difference in the measured $s(x, Q)$, compared to that obtained from our global analysis, lies mainly in the small $x$ region. We have been informed by the CCFR collaboration that preliminary results from a next-to-leading order analysis of the same data do not lead to substantial changes in these results. If this measurement of the strange sea is correct, then the difference of the neutrino and muon $F_{2}$ structure functions shown in Fig. 2 cannot be fully attributed to the strange quark. This suggests either additional sources of theoretical uncertainties so far neglected in all global analyses, or an incompatibility of the $F_{2}$ measurements of the CCFR and NMC experiments within the stated errors in the very small $x$ region. To see how changes in experimental systematic errors might affect the situation, we have performed new fits fixing $\kappa=0.5$ and increasing the experimental systematic error in the smallest- $x$ bins by a multiplicative factor. Reasonable fits with $\chi^{2}$ in-between the best CTEQ fits and the " $\kappa 5$ fit" (cf. Table II) are obtained with a factor of order 2 for either of the experiments. Aside from readjustments among the sea-quark flavors, the shapes of the other parton distributions remain quite stable. Details will be described elsewhere.

It is important to emphasize that the question about the strange quark should not obscure the main results of this paper: the determination of a new generation of parton distributions based on recent highly precise experimental measurements and phenomenological advances. The progress made is evident in the $\chi^{2}$ comparison between Tables I and II. The new degree of accuracy is also responsible for bringing the potential conflict between the new $F_{2}$ measurements and the dimuon results to light. To resolve the current puzzle we need to reevaluate all theoretical uncertainties; improve the treatment of systematic errors in the global analysis; and, finally, include the dimuon data in the analysis. These efforts are underway. At the same time, we urge the key experiments to re-examine their data in the small- $x$ region to see if our observed discrepancy persists under closer scrutiny.

We thank various members of the CCFR Collaboration, particularly Sanjib Mishra and Michael Shaevitz for in-depth discussions on the strange quark issue. 


\begin{tabular}{|c|c||ccccccccc|}
\hline & Total $\chi^{2}$ & $\operatorname{ccf} F_{2}$ & $\mathrm{ccf} F_{3}$ & nmcH & nmcD & nmcR & bcdH & bcdD & E605 & Dir $\gamma$ \\
\hline KMRS B0 & 3954 & 22.16 & 3.34 & 6.11 & 8.46 & 1.29 & 1.25 & 1.40 & 1.20 & 1.02 \\
MT S1 & 3786 & 21.56 & 4.32 & 4.43 & 6.38 & 1.81 & 1.57 & 1.44 & 1.00 & 1.77 \\
MT B2 & 1415 & 3.10 & 3.14 & 2.21 & 1.41 & 1.27 & 0.76 & 1.05 & 0.90 & 2.46 \\
\hline \hline MRS D0 & 1643 & 5.31 & 3.71 & 1.47 & 1.24 & 1.27 & 1.17 & 1.21 & 1.16 & 0.96 \\
\hline
\end{tabular}

Table I: $\chi^{2}$ of some widely used parton distributions applied to current experimental data. The first column gives the total $\chi^{2}$ for 917 data points (all experiments). The other columns give the $\chi^{2}$ per data point for the individual data sets: $C C F R F_{2}$ and $F_{3}$; $N M C H, D$, and $F_{2}^{n} / F_{2}^{p} ; B C D M S H$ and $D ; E 605$; and the combined direct photon production data ( $W A 70, E 706$ and $U A 6)$.

\begin{tabular}{|c|c||ccccccccc|}
\hline & Total $\chi^{2}$ & $\mathrm{ccf} F_{2}$ & $\mathrm{ccf} F_{3}$ & $\mathrm{nmcH}$ & $\mathrm{nmcD}$ & $\mathrm{nmcR}$ & $\mathrm{bcdH}$ & $\mathrm{bcdD}$ & $\mathrm{E} 605$ & Dir $\gamma$ \\
\hline \# pts. & 917 & 77 & 77 & 83 & 83 & 89 & 168 & 156 & 119 & 47 \\
\hline CTEQ1M & 860 & 0.68 & 0.71 & 1.25 & 0.90 & 1.34 & 0.72 & 1.12 & 0.87 & 0.73 \\
CTEQ1D & 861 & 0.74 & 0.90 & 1.26 & 0.91 & 1.30 & 0.65 & 1.07 & 0.83 & 0.70 \\
CTEQ1MS & 861 & 0.71 & 0.79 & 1.26 & 0.89 & 1.25 & 0.71 & 1.10 & 0.87 & 0.74 \\
\hline \hline CTEQ1ML & 892 & 0.78 & 0.80 & 1.24 & 0.86 & 1.28 & 0.82 & 1.18 & 0.83 & 0.75 \\
CTEQ1L & 914 & 0.89 & 0.86 & 1.03 & 1.07 & 1.50 & 0.68 & 0.99 & 1.15 & 0.73 \\
\hline \hline M2 & 912 & 0.81 & 0.96 & 1.28 & 0.87 & 1.30 & 0.76 & 1.11 & 0.99 & 0.89 \\
$\kappa 5$ & 948 & 0.96 & 0.87 & 1.26 & 1.00 & 1.28 & 0.72 & 1.14 & 1.09 & 0.86 \\
\hline
\end{tabular}

Table II: $\chi^{2}$ of various $C T E Q$ global fits to current data. Column labels are the same as in Table I. The number of fitting parameters (parton distribution shape and relative experimental normalization) is of the order of 30 to 35; resulting in about 880 degrees of freedom. 


\section{Figure Captions}

Fig. 1 : Sea-quark distributions. The flavor labels on the left are for CTEQ1M; those on the right for MRS_D0.

Fig. 2 : Comparison of experimentally measured $\frac{5}{6} F_{2}^{\nu d}-3 F_{2}^{\mu d}$ with $x s\left(x, Q_{0}\right)$ from two of our fits, CTEQ1M and K5, and from MRS_D0. Errors of the two experiments are added in quadrature and superimposed on the relevant curve to indicate the size of the experimental uncertainty.

\section{References}

[1] J. Collins, D. Soper and G. Sterman in Perturbative QCD, Ed. A. Mueller, World Scientific (1989).

[2] D. Duke and J. Owens, Phys. Rev. D30, 49 (1984).

[3] J.G. Morfin and W.K. Tung, Z. Phys. C52 13, (1991).

[4] For a review and detailed references, see J.F. Owens and W.K. Tung, "Parton Distributions of Hadrons" in Ann. Rev. Nucl. Sci. (1992).

[5] NMC Collaboration, P.Amaudruz et al, Preprint CERN-PPE/92-124.

[6] CCFR Collaboration, Nevis Preprints, S.R.Mishra et al., \#1459; W.C.Leung et al., \#1460; and P.Z.Quintas et al., \#1461.

[7] J. Kwiecinski, A.D. Martin, R.G. Roberts and W.J. Stirling, Phys. Rev., D42, 3645 (1990)

[8] A. Martin, R. Roberts, and J. Stirling, Durham and Rutherford Preprint (DTP/92/16, RAL-92-021)

[9] We used an emperical formula based on NMC measurements at small $x$ and SLAC measurements at large $x$. (Private communication from A. Bruell (NMC).

[10] Wu-Ki Tung in Proceedings of 1992 International Conference on High Energy Physics, Dallas (to be published).

[11] See Wu-Ki Tung, in Proceedings of Workshop on Hadron Structure Functions and Parton Distributions, Ed. Geesaman et.al., Singapore:World Scientific, (1990).

[12] A. C. Benvenuti et.al., Phys. Lett., 223B 490 (1989); P. Z. Quintas et.al. in [11 and in [6].

[13] P.N. Harriman, A.D. Martin, R.G. Roberts \& W.J. Stirling, Phys. Rev. D42, 798 (1990). 
[14] See, for instance, H. Abramowicz et.al., Z. Phys., C15, 19 (1982); K. Lang et.al., Z. Phys. C33, 483 (1987); M. Shaevitz, in [10]

[15] M.G. Aivazis, F. Olness \& Wu-Ki Tung, Phys. Rev. Lett. 65, 2339 (1990).

[16] P.L. McGaughey et.al., Phys. Rev. Lett. 69, 1726 (1992).

[17] B. Badelek \& Jan Kwiecinski, Nucl.Phys., B370, 278 (1992); V.R. Zoller Z.Phys., C54, 425 (1992); M. Strikman in [10].

[18] See Ref.[15]; and J.J. van der Bij \& G.J. van Oldenborgh, Z. Phys. C51 477 (1991).

[19] Proceedings of the Workshop Physics at HERA, Ed. W. Buchmuller and G. Ingelman, DESY, 1991. 